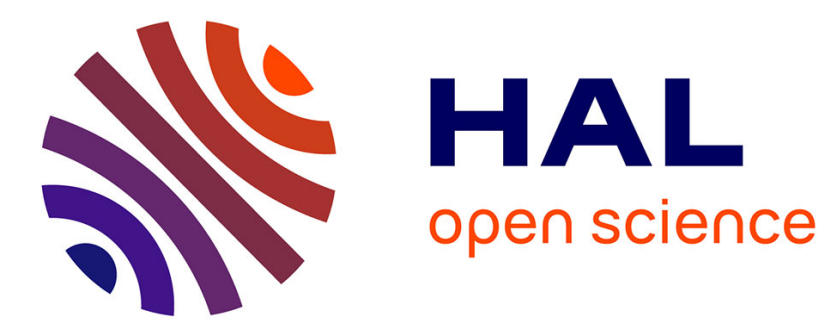

\title{
Total Memory Optimiser: Proof of concept and compromises
}

Maurice Clerc

\section{- To cite this version:}

| Maurice Clerc. Total Memory Optimiser: Proof of concept and compromises. 2016. hal-01294101

\section{HAL Id: hal-01294101 \\ https://hal.science/hal-01294101}

Preprint submitted on 27 Mar 2016

HAL is a multi-disciplinary open access archive for the deposit and dissemination of scientific research documents, whether they are published or not. The documents may come from teaching and research institutions in France or abroad, or from public or private research centers.
L'archive ouverte pluridisciplinaire HAL, est destinée au dépôt et à la diffusion de documents scientifiques de niveau recherche, publiés ou non, émanant des établissements d'enseignement et de recherche français ou étrangers, des laboratoires publics ou privés. 


\title{
Total Memory Optimiser: Proof of concept and compromises
}

\author{
Maurice Clerc (Maurice.Clerc@WriteMe.com)
}

26th March 2016

\begin{abstract}
For most usual optimisation problems, the Nearer is Better assumption is true (in probability), This property is taken into account by the classical iterative algorithms, either explicitly or implicitly, by forgetting some information collected during the process, assuming it is not useful any more. However, when the property is not globally true, i.e. for deceptive problems, it may be necessary to keep all the sampled points and their values, and to exploit this increasing amount of information. Such a basic Total Memory Optimiser is presented. We show on an example that it can outperform classical methods on deceptive problems. As it is very computing time consuming as soon as the dimension of the problem increases, a few compromises are suggested to speed it up.
\end{abstract}

\section{Motivations}

Today (2016-03) all iterative optimisers do forget some positions they have previously sampled in the search space, sooner or later, even a method like Tabu Search [Glover and Laguna(1997)]. This is a lost of information about the "shape" of the landscape of the problem at hand. An obvious drawback is that the same position may be sampled and evaluated several times, which is useless. In order to prevent this undesirable behaviour, the classical algorithms progressively define "bad areas" in which the probability to be sampled is null or extremely small.

On the one hand, there is a risk to be wrong by doing that, but, on the other hand, there is no doubt that most of these methods are efficient in practice on many problems. We claim here that it is because for these problems a "Nearer is Better in probability" assumption is valid.

But what if it is not true? In such a case, it may be useful to make use of an algorithm that takes into account all the information that is collected during the iterative search. 


\section{Nearer is Better assumption}

The NisB assumption has been studied in [Clerc(2007)] and [Clerc(2015)]. Let us just give here a short definition and a few examples.

\subsection{Definition}

What we call here the NisB correlation can be estimated as follows:

- sample at random (uniform distribution) $N$ times three positions and sort them so that $f(x)_{1} \leq f(x)_{2} \leq f\left(x_{3}\right)$;

- let $n_{\text {is } B}$ be the number of times we have $f(x)_{1} \leq f(x)_{2}<f\left(x_{3}\right)$ and distance $\left(x_{1}, x_{2}\right)<\operatorname{distance}\left(x_{1}, x_{3}\right)$.

- then the correlation is defined so that it is in $[-1,1]$ by

$$
\rho=-1+2 \frac{n_{i s B}}{N}
$$

\subsection{Examples}

For a strictly monotonic function, the NisB correlation is of course equal to 1 . For most usual problems it is positive. However, as we can see in the table 1 it can easily be negative, as soon as there are some plateaus ${ }^{1}$. The precise definitions of these four problems are in the Appendix.

Also, if it is not equal to 1 , it means that it is sometimes locally negative. For example, for the Parabola of the figure 1 the global correlation is $17 / 18=0.944$, not equal to 1 because of triplets of points "around" the minimum.

Actually, it may be interesting to evaluate the correlation as a function of the vicinity. Here, we define this vicinity as a proportion of the search space around each point. So, the global correlation is given for a vicinity equal to 1 . As intuitively expected, the smaller the vicinity, the higher the correlation, but not a lot, and not even always (see the Flash example).

\section{Basic Total Memory Optimiser}

We now define a Total Memory Optimiser, just a basic one for the moment, in the hope it can outperform usual methods on deceptive problems, i.e. the ones with negative NisB correlation. At first glance, according to the figure 3, the components of this algorithm seem quite classical. However, by examining them in details we will see some important specific features.

\footnotetext{
${ }^{1}$ This is an open question: is it possible to define a lipschitzian function without any plateau but with a negative NisB correlation?
} 


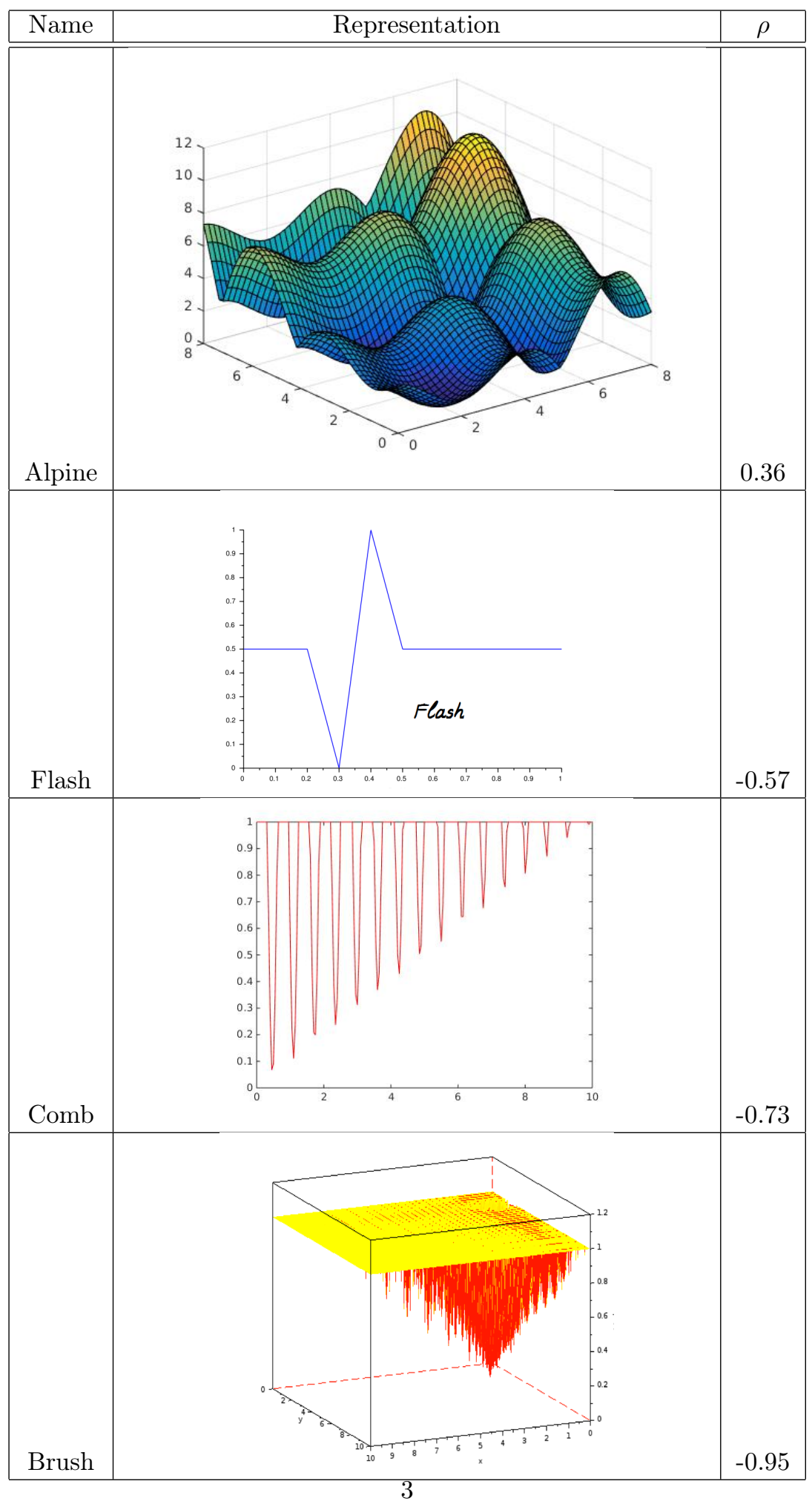

Table 1: Examples of Nearer is Better correlation. 


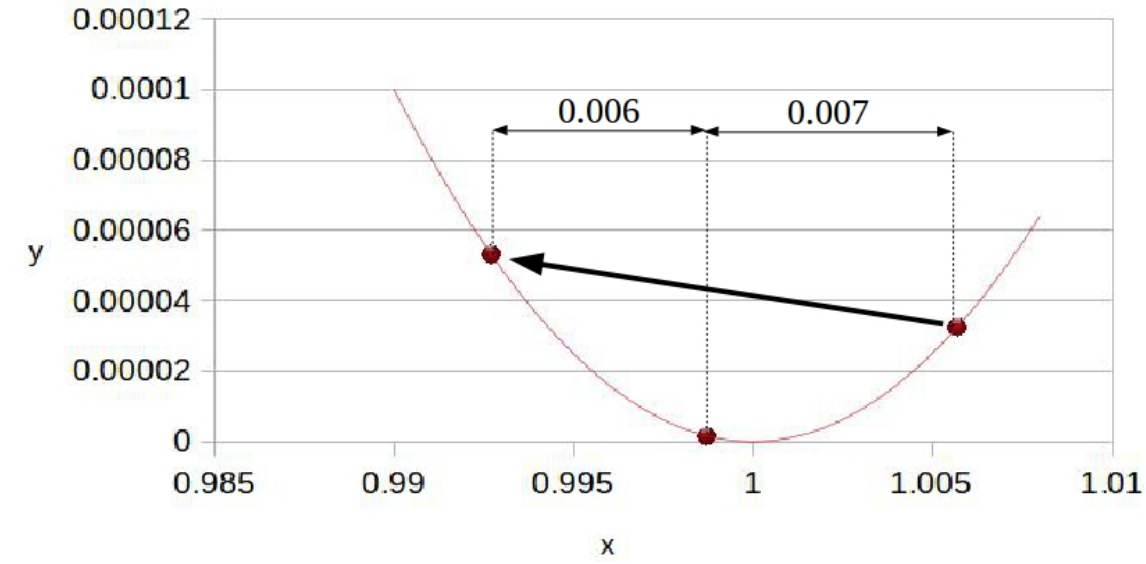

Figure 1: When Nearer is Worse, locally. However, globally, the correlation is positive.

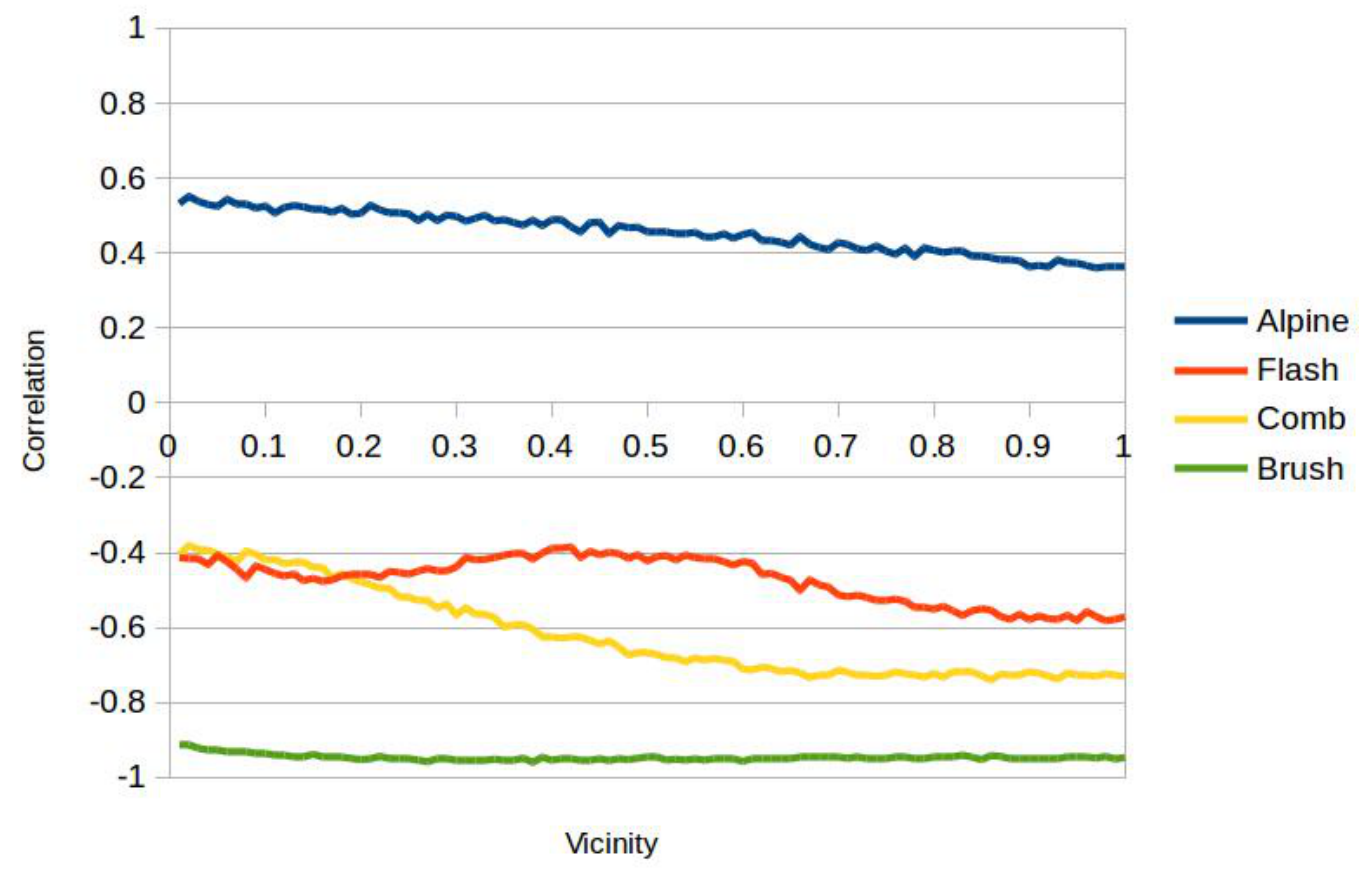

Figure 2: NisB correlation vs Vicinity. 


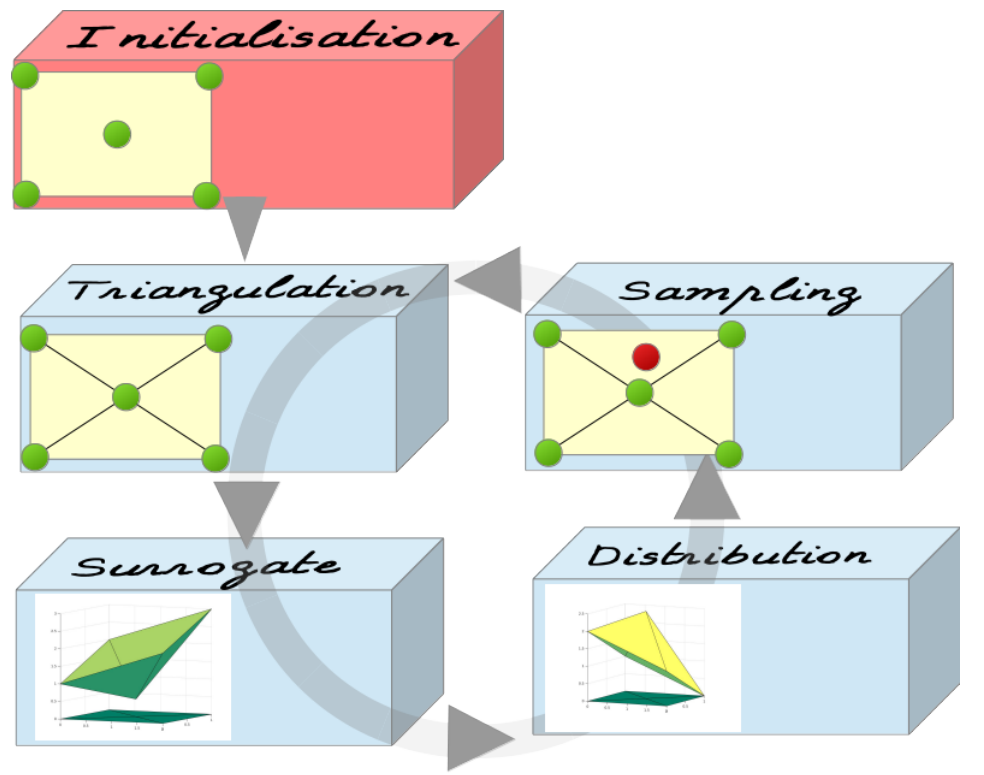

Figure 3: Basic Total Memory Optimiser.

\subsection{Initialisation (first samples)}

At the very beginning we know nothing, except the search space. It is then easy to prove that the first point to sample must be the centre of this search space, in order to minimise the risk to be wrong (see the Appendix).

For the other points, we have to anticipate a bit the next step, which is triangulation. We want that the triangulation is able to cover the whole search space, so that, in principle, any point may be then sampled. As the search space is supposed to be a convex polyhedron, a sure way to do that is to sample all the corners. In practice the search space is a $D$-rectangle (which can easily be transformed into a $D$-square, in order to later simplify strategies like "search around"). So, finally, the number of initial points is $2^{D}+1$.

Of course, it increases very rapidly with $D$, and it may be unacceptable for small computers. But for the moment, to describe this basic TMO, we do not care. We will see later some possible compromises.

\subsection{Cycle: From Sampling to Triangulation}

At a given time step we have sampled $N$ points. We will explain in the next step that the surrogate function is made of "triangular" facets (interval for $D=1$, real triangles for $D=2$, tetrahedron for $D=3$, etc.), whose projections on the search space are of course also "triangles". In order to have no overlapping of these "triangles" an elegant way is to define a Delaunay's triangulation between the $N$ points. Actually, this is a simplified variant of the approach described in 


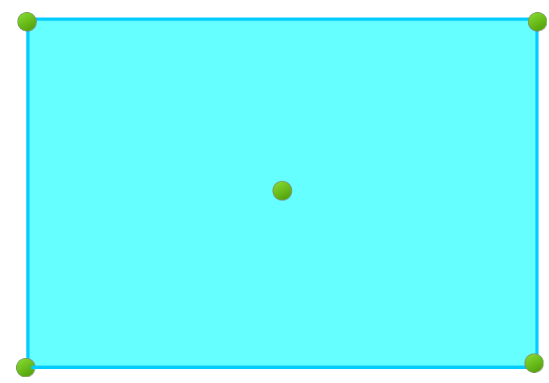

Figure 4: Initialisation: centre + corners.

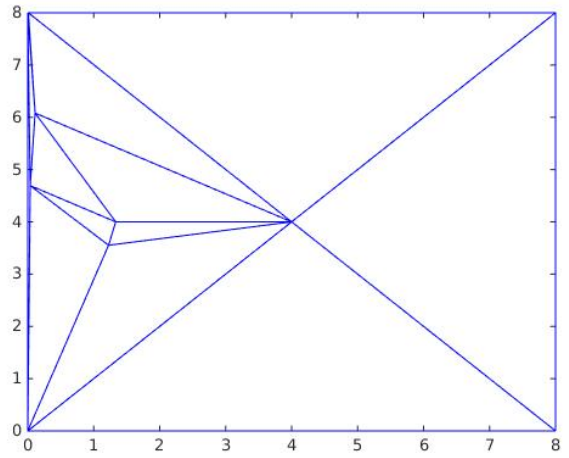

(a) 10 points

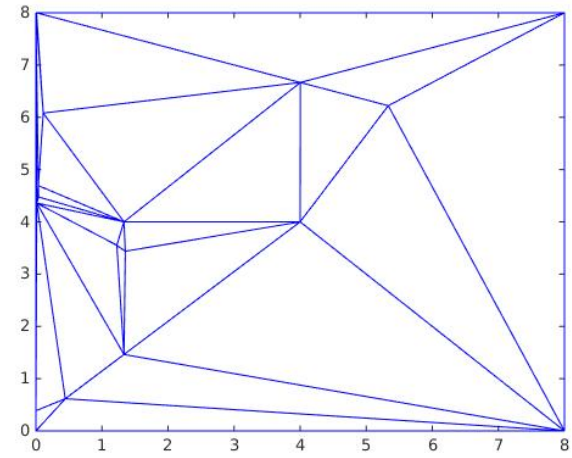

(b) 20 points

Figure 5: Delaunay's triangulations (Alpine problem, solution point on $(1,2)$ ).

[Beyhaghi et al.(2015)Beyhaghi, Cavaglieri, and Bewley].

The figure 5 shows two such triangulations, coming from the Alpine problem that is described in the Appendix.

Again, this method is very costly when the dimension increases, again we do not care for the moment, and, again, there are some possible cheaper compromises.

\subsection{Cycle: From Triangulation to Surrogate function}

Using surrogate functions is not at all new. See for example [Han and Zhang(2012)]. However, here, we modify it after each new sampling and, moreover, we want to use the best one under as few hypotheses as possible. Indeed, most "aesthetic" surrogate functions do suppose some superfluous properties like differentiability everywhere, at least implicitly.

To simplify the reasoning, let us consider a 1D problem whose value space is $\left[y_{\min }, y_{\max }\right]$, two sampled points $x_{1}, x_{2}$, and their values $f\left(x_{1}\right), f\left(x_{2}\right)$. We 
do not know the values of $f$ between the two points, and we want to estimate them by using the surrogate function $g$ that minimises the risk to be wrong, i.e. the risk to sample $x$ with $g(x) \neq f(x)$. We must have $g\left(x_{1}\right)=f\left(x_{1}\right)$ and $g\left(x_{2}\right)=f\left(x_{2}\right)$, but for any other point $x$ in $] x_{1}, x_{2}$ [ we just know that we must have $y_{\min } \leq g(x) \leq y_{\max }$. If we do not make any hypothesis, it is easy to prove that the best $g$ is given by $g(x)=\frac{y_{\min }+y_{\max }}{2}$ (see the figure 6a and the Appendix). This is not satisfying for this plateau function does not increase the quantity of information (even if only guessed) we can use.

So, we do make a hypothesis. Here, it is that the NisB correlation between the two points is locally positive ${ }^{2}$. It implies that $g$ must be strictly monotonic. But there is still an infinity of possible functions (figure 6b). So, we also apply the Occam's razor, or, more formally, we assume that the variability (see the Appendix for a formal definition) of the function must be minimal. Then, the only possible function is the linear one. More generally, on $D$ dimensions problems, the surrogate function is made of "triangular" facets (see figures 6c and $6 \mathrm{~d})$.

\subsection{Cycle: From Surrogate function to Estimation of Dis- tribution}

This step is of course crucial. TMO is stochastic, and therefore it has to make use of an estimation of distribution, as all stochastic methods do, either implicitly or explicitly. Here, it is explicitly.

Let us consider the surrogate function of the figure 6c. It contains all the information collected during the iteration: the sure one (sampled points), and the probable one (interpolations). Let us suppose first the problem is a maximisation. The high values of the surrogate function tell us where probably are the high values of the true function. So, it seems reasonable (still because the local NisB principle) to more probably sample "around" the points of high value, and conversely less probably "around" the points of low value.

It means that the probability distribution should have the same "shape" as the surrogate function (see figure 7a). And in case of minimisation, this is the contrary: it should have an opposite shape (see figure $7 \mathrm{~b}$ ).

So, we have a straight way to define the estimation of distribution: just transforming the surrogate function into a probability distribution, either directly, or by taking its opposite (in practice by applying a formula like minimum + maximum $-g$ ).

Note that it is perfectly possible to "distort" the distribution thanks to a userdefined morphing parameter, either optimistic or pessimistic (see the Appendix). To keep the presentation simple, we will not do that in the examples.

\footnotetext{
${ }^{2}$ It may seems contradictory with the fact that we want to cope with problems for which the NisB correlation is globally negative. Even in such a case, it is sometimes locally positive, and more and more when the number of points increases.
} 


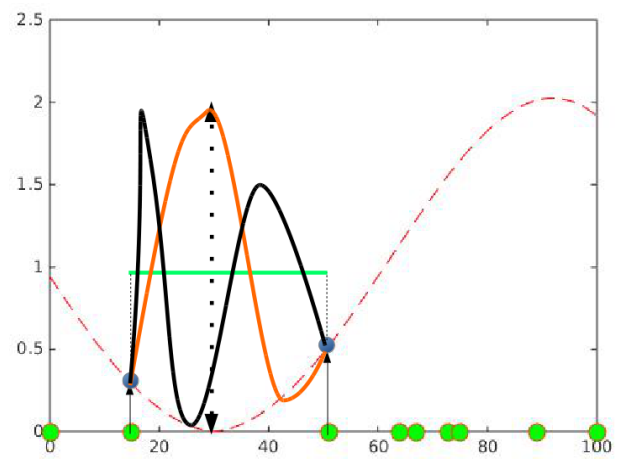

(a) No hypothesis. The best surrogate function is a plateau.

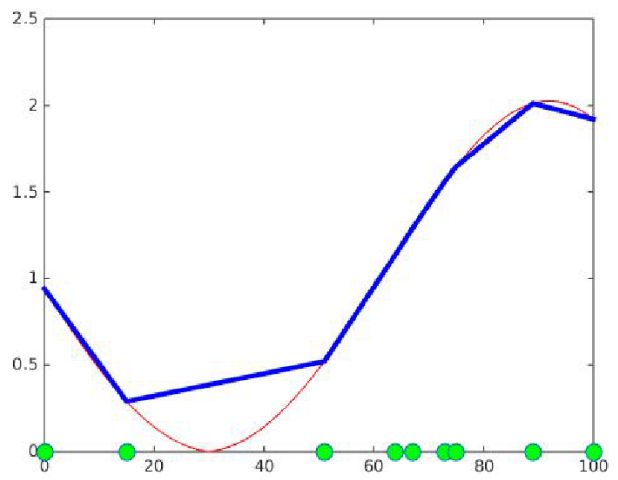

(c) Piece-wise linear surrogate function (1D).

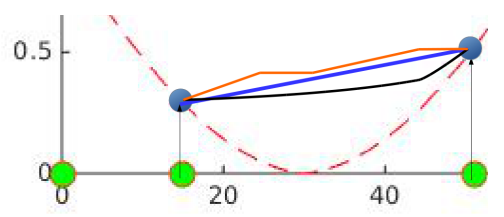

(b) NisB correlation locally positive, and minimal variability.

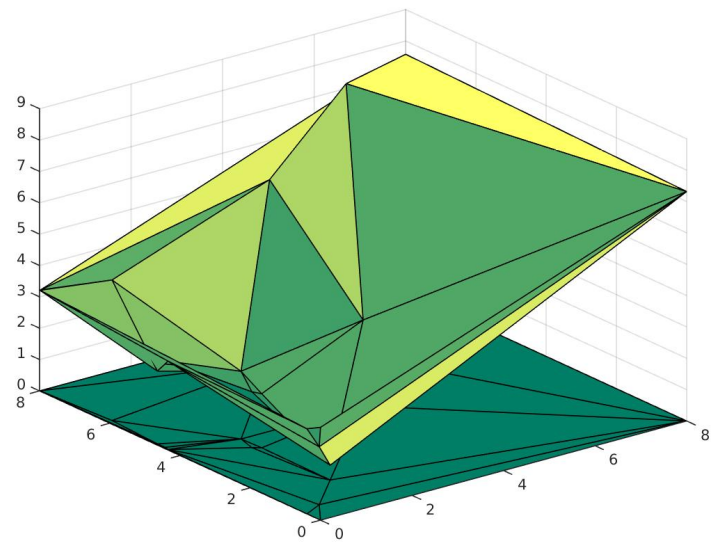

(d) Piece-wise linear surrogate function (2D).

Figure 6: Choosing the "best" surrogate function. Without any hypothesis, it is a plateau. With the locally positive NisB correlation hypothesis, it is monotonic. And with the minimum variability constraint, it is a piece-wise linear one. 


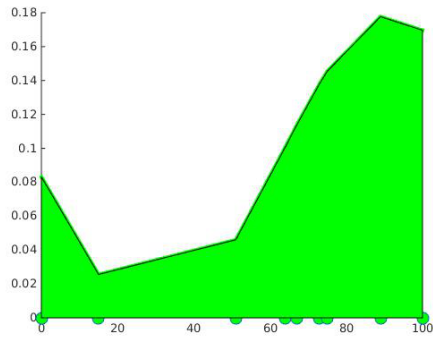

(a) For maximisation

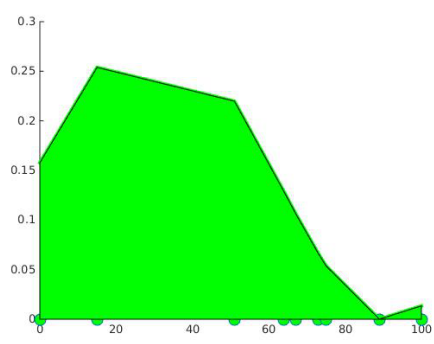

(b) For minimisation

Figure 7: Estimations of distribution, derived from the surrogate function of the figure $6 \mathrm{c}$.

\subsection{Cycle: From Estimation of Distribution to Sampling}

This step is a classical one. Moreover, as the distribution is entirely defined by the sampled points, it is technically easy. In order to improve the efficiency of TMO, we also make use of "representatives" of the triangular facets. A representative can for example be:

1. the centre of the facet;

2. a weighted gravity centre (weights depending on the values of the vertices);

3. a random point inside the facet.

In what follows, we simply use the method 1 (see the figure 8). We virtually put all the values (of the sure points and of the representatives) along a line, side by side, draw at random a number (uniform distribution) between 0 and their sum, and go back to the corresponding value.

If it is a representative, we indeed sample this new point. If it is an already known point $x_{1}$, we sample "around" it. In practice, the search space has been normalised as a $D$-square (if $D \geq 2$ ), and "around" means "inside a small $D$ square centred on the known point". If $D=1$, we of course just consider intervals.

In the examples, "small" is defined as follows:

- find the nearest known point $x_{2}$;

- the edge of the $D$-square is $2 \alpha\left\|x_{1}-x_{2}\right\|$, where $\alpha$ is a user-defined parameter. In the examples of this paper $\alpha=\frac{1}{3}$.

Note that instead of using the $g(x)$ values it is perfectly possible to sort them and to use their ranks. Actually some experiments suggest that it may be a better way, and this is what is done here. 


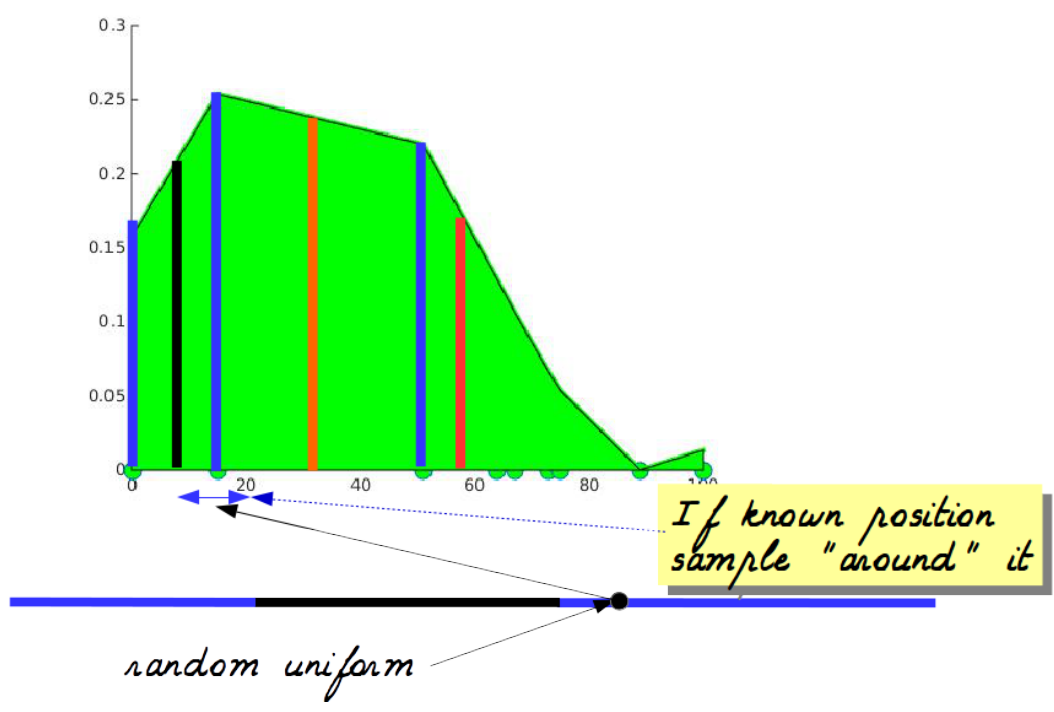

Figure 8: Sampling from the estimation of distribution.

\section{Compromises}

As soon as the dimension of the problem increases, it is clear that the computing cost becomes rapidly unacceptable in practice, as we can see on the figure 9 .

So it is useful to study some compromises. An easy first one is to replace the initial set of $2^{D}+1$ positions by a smaller one. Keeping the centre of the search space is costless, but for the other initial points we may for example:

- sample at random $N$ points (a very usual method, in fact);

- sample at random $N$ points and $C$ corners (typically $C=1$ ).

It means that the "covering" of the search space by triangulation is now just a partial one, but if we launch several runs, we hope that the solution will be inside a triangle, and has a non null probability to be found. Actually, it is the way classical optimisers do work.

The complete Delaunay's re-triangulation after each new sampled point is a costly process. We can replace it by a local re-triangulation. A very simple one is shown on the figure 10. We just subdivide the "triangle" in which is the new point.

\section{Comparisons}

We can now make some comparisons between TMO (basic and some variants with compromises) and a few classical optimisers. For fair comparisons, we have to carefully specify two things: 


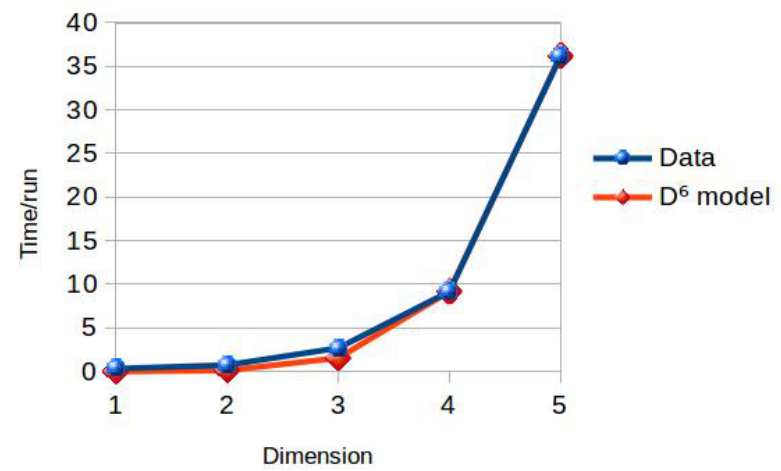

Figure 9: Alpine function. Computing time vs dimension.

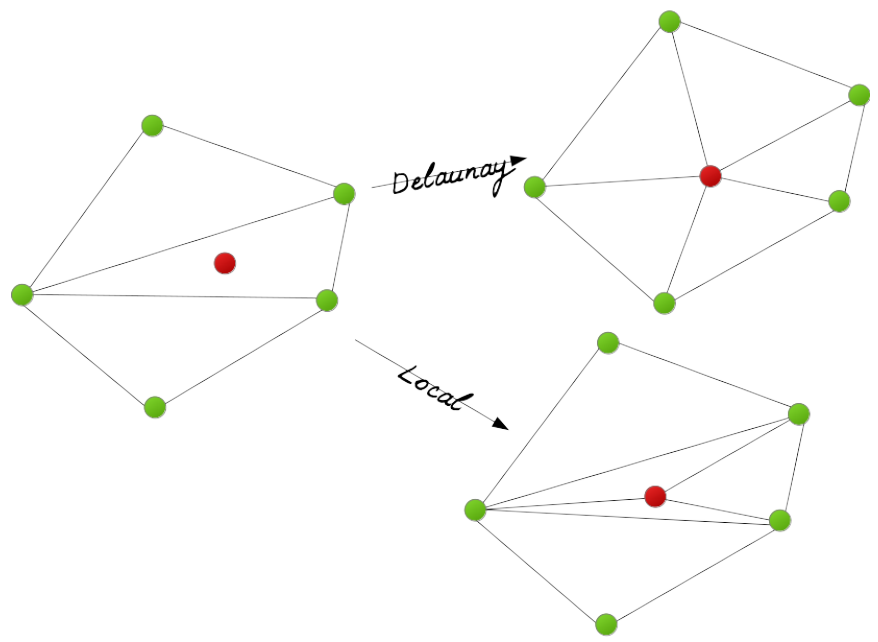

Figure 10: Simplifying the re-triangulation. 
- the budget;

- the user's demand.

\subsection{Budget}

A budget for iterative optimisation on a computer has at least three components:

- a maximum number of evaluations $E$;

- a maximum computing time $T$;

- a maximum memory size $M$.

In this paper, we want to check how efficient is TMO in taking into account all the information collected during the iterative process. So, for comparisons, we will just use the same $E$ for several methods. Actually, this is a classical approach.

\subsection{Demand}

Many studies take as comparison criterion the mean result over $n$ runs, or the median value, and perform statistical analyses to estimate in which extend such values are reliable. But in practice a user does not care of means or medians. His/her question is (in case of minimisation): "With this budget and this algorithm, what is the probability to get a result smaller than $\varepsilon$ ".

It is out of the scope of this paper to analyse in details how to spend a given budget. We just apply here the classical method: $n$ independent runs of $E / n$ evaluations. But keep in mind this is far to always be the best way.

To answer the user's question, we need to estimate the probability distribution of the results. In practice, it means we need to build an estimation of the PDF (Probability distribution function) or, easier to use, an estimation of the CDF (Cumulative Distribution Function). By doing that, when comparing two algorithms $A_{1}$ and $A_{2}$, it often happens that the CDF curves have at least one cross point, for a given result $\varepsilon^{*}$. Let us suppose $\operatorname{CDF}\left(A_{1}\right)$ is "above" $\operatorname{CDF}\left(A_{2}\right)$ for results smaller than $\varepsilon^{*}$, and the contrary for higher values. If the user is very demanding (i.e. does accept only values smaller than $\varepsilon_{\max } \leq \varepsilon^{*}$, then $A_{1}$ is the best choice. But if the user accepts values smaller than $\varepsilon_{\max }>\varepsilon^{*}$, then, on the contrary, $A_{2}$ is the best choice.

\subsection{Some results}

We first compare a few TMO variants, with some compromises, and the basic TMO. As we will see, some variants may be better than this basic TMO. But to better highlight the specificity of TMO, i.e. its efficiency on problems of negative global NisB correlation, we will compare then three classical methods only to the basic TMO. 


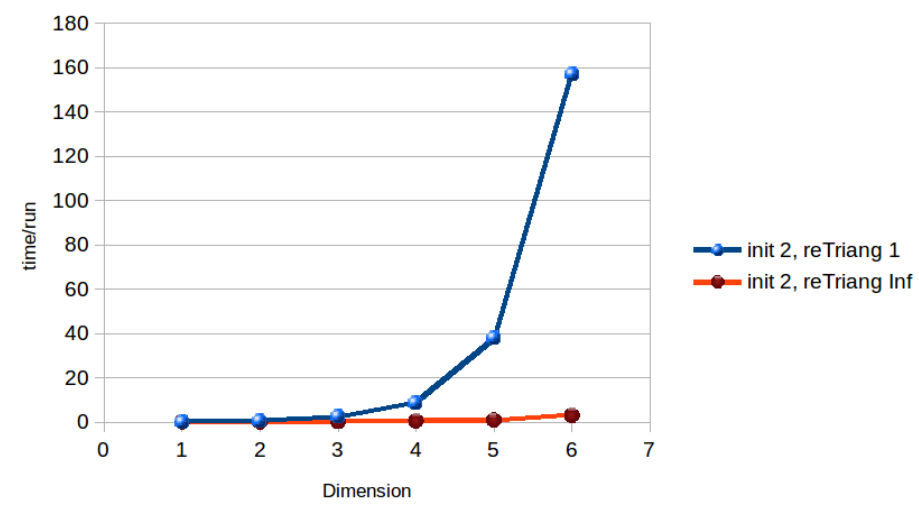

Figure 11: Using simplified retriangulation dramatically reduces the computing time (Alpine, 150 evaluations).

\subsubsection{TMO variants}

On the figure 12, "init 2" is for the initialisation method of the basic TMO (centre + corners), and "init 0 " is for centre + random initialisation (here with 89 positions). And "reTriang $k$ " means Delaunay's re-triangulation every $k$ new positions, local simplified re-triangulation else. In particular "reTriang Inf" means Delaunay's triangulation just once, after initialisation, and then only the simplified method is used. The figure 11 shows that the computing time is easily then far smaller.

Moreover, at least for problem with a positive NisB correlation like Alpine, not only complete and systematic re-triangulation is not necessary, but partial ones may give better results: higher probability for small values, as we can see on the figure 12 .

When the NisB correlation is negative, as for the Brush function, we have something similar. On the figure 13 the three curves with "init 0" are better than the ones with "init 2". Note that, though, we are here in a case where the number of corners is far smaller than the number of random initial points. The conclusion may not be valid for dimension greater than 6 . Unfortunately, the laptop used for this study can not cope with Delaunay's triangulations in dimension 7 or greater.

So, we now consider only the basic TMO for comparisons with classical methods, i.e "init 2, reTriang 1".

\subsubsection{TMO vs others}

The figure 14 represents the CDFs of four methods on the Alpine function. As said, the first one is the basic TMO, and the other ones are GA-MPC ${ }^{3}$, CMA-ES

\footnotetext{
${ }^{3}$ We would like to thank Dr. Saber Elsayed for providing the MATLAB ${ }^{\complement}$ code of GAMPC.
} 


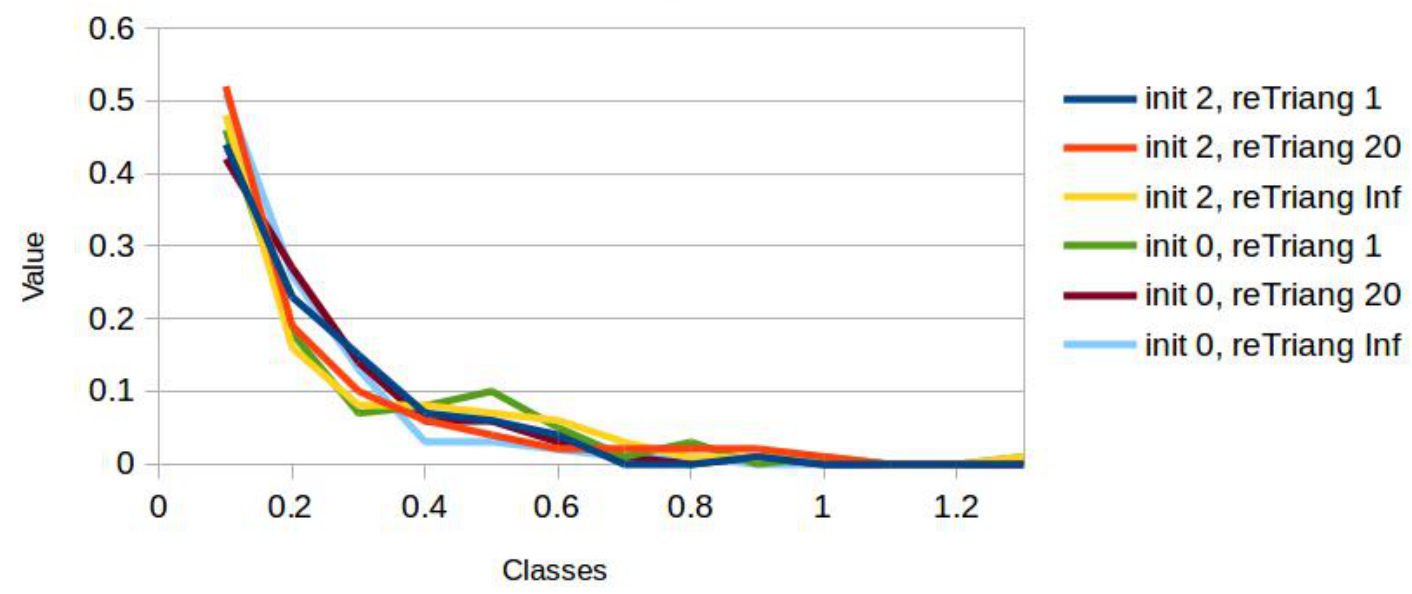

Figure 12: Alpine, TMO variants, 100 runs of 150 evaluations, PDFs curves.

[Hansen(2009)] , and APS 11. GA-MPC [Elsayed et al.(2011)Elsayed, Sarker, and Essam] was the winner of the CEC 2011 competition, CMA-ES the winner of the CEC 2005 competition (in fact, we used a more recent and better version 3.62, downloaded from https://www.lri.fr $/{ }^{\sim}$ hansen/cmaes_inmatlab.html). For APS [Omran and Clerc(2013)], a well-documented MATLAB ${ }^{\circledR}$ implementation can be downloaded from http://aps-optim.info. It easily outperforms both GAMPC and CMA-ES on the CEC 2011 problems, but the point here is that TMO is clearly the worst method on Alpine, unless results greater than 0.4 are acceptable: in that case it outperforms CMA-ES (but still not the other ones).

The conclusions are different on a very deceptive function like Brush (see the figure 15). Now TMO is the best choice as soon as we accept only good (small) results. Note that on such a problem CMA-ES is particularly bad. This is probably because it assumes more than the other methods that the NisB assumption holds, and is also more biased, as we can see on the signatures (see the Appendix).

\section{When and how using TMO?}

In this study we have given a precise definition of "deceptive problems", by saying there are the ones for which the global NisB correlation is negative. However, in the field of optimisation, "deceptive" usually just means "very difficult". In particular, combinatorial problems a often said to be defective.

Preliminary tests show that for many combinatorial problems, if not all, the NisB correlation is positive. Of course, this is done by using a specific distance, for example the Kendall tau one's, or, in a more intuitive way, the minimum number of transpositions to transform a given "position" (typically a 


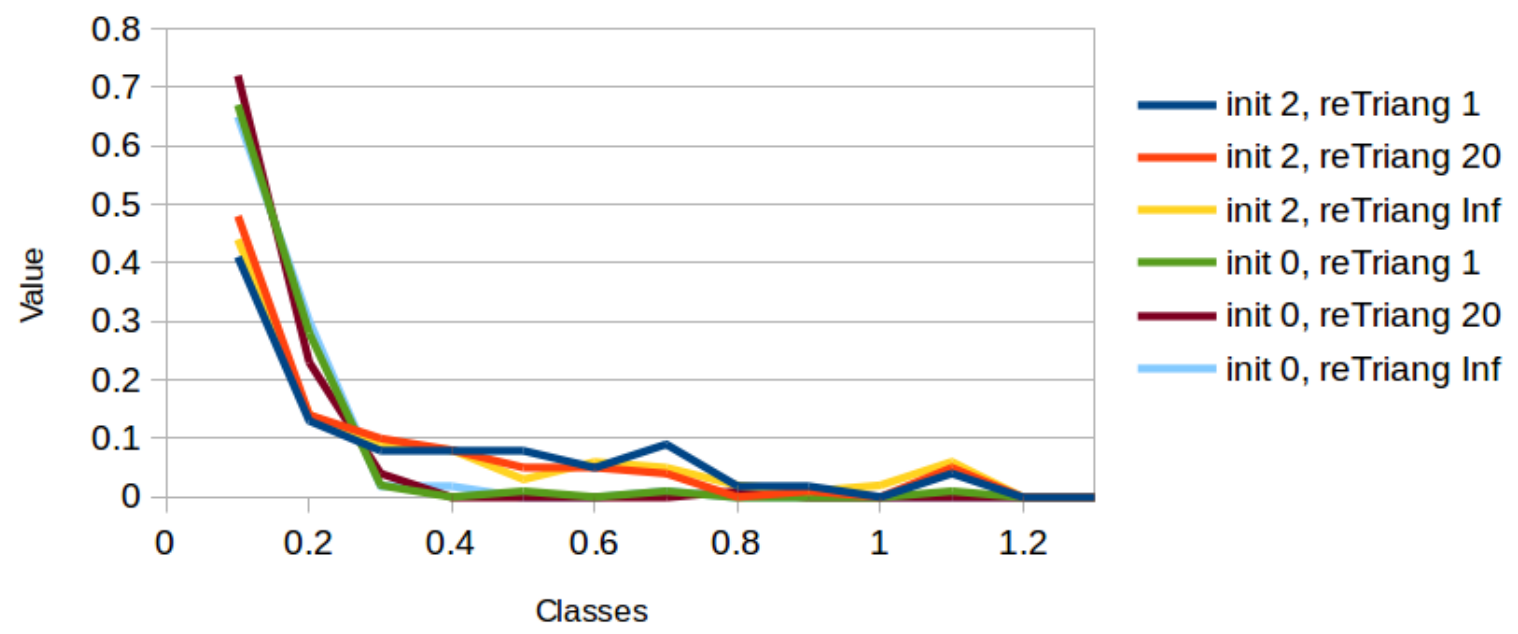

Figure 13: Brush, TMO variants, 100 runs of 150 evaluations, PDFs curves.

permutation of $K$ elements) into another one.

On the one hand, we have said that TMO is for problems with negative NisB correlation, so it is probably not a good tool for combinatorial problems. On the other hand, for these problems, as the correlation is just slightly positive it may be nevertheless worth trying to apply it.

Also, TMO could be combined with a classical iterative algorithm A, by using the following strategy:

- compute the NisB correlation after each new evaluation;

- if positive, use A;

- if negative, use TMO.

Again, nothing proves it will work, but, again, it seems worth trying.

If it appears that TMO, either alone or in combination, indeed gives good results on some real world problems, it will be necessary to increase its computational efficiency. We already have seen a few possible ways, but more are possible, and should be tested.

\section{Appendix}

\section{Problem definitions}

\section{Alpine}

For dimension $D$, the search space is $[0,4 D]^{D}$. Function $f$ is defined as: 


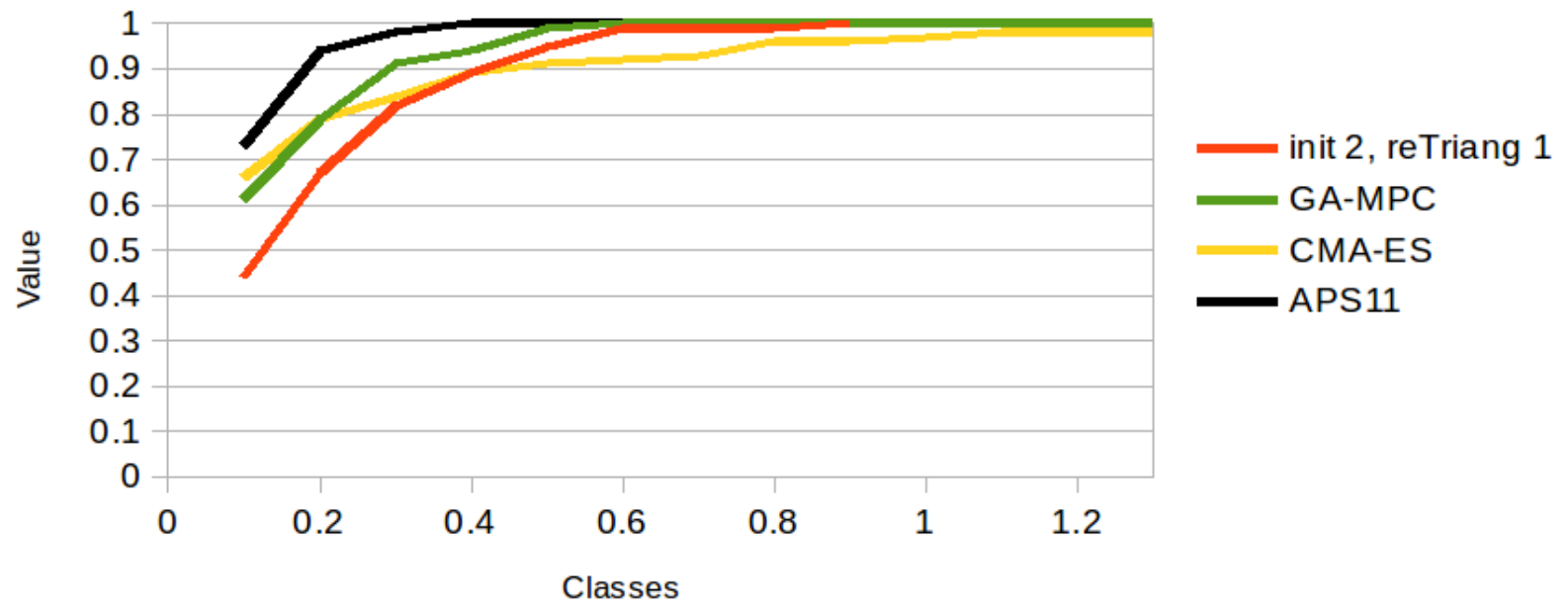

Figure 14: Alpine, TMO vs others, 100 runs of 150 evaluations, CDFs curves. On this "positive" NisB problem, TMO is the worst method, unless the user is not very demanding, accepting results greater than 0.4. In that case, CMA-ES is the worst.

$$
f\left(x_{1}, \ldots, x_{D}\right)=\sum_{d=1}^{D}\left|x_{d, \delta} \sin \left(x_{d, \delta}\right)\right|+0.1 x_{d, \delta}
$$

with $x_{d, \delta}=x_{d}-\delta d$. In this case, we have simply chosen $\delta=1$. This parameter serves to ensure that the minimum is not at the center of the search space or on a diagonal. The problem is multimodal and non-separable.

\section{Deceptive 1 (Flash)}

The search space is $[0,1]$. Function $f$ is defined as:

$$
\left\{\begin{array}{cl}
x \leq 2 c_{1} & \rightarrow f(x)=c_{2} \\
2 c 1<x \leq 3 c_{1} & \rightarrow f(x)=c_{2}-\frac{c_{2}}{c_{1}}\left(x-2 c_{1}\right) \\
3 c_{1}<x \leq 4 c_{1} & \rightarrow f(x)=\frac{2 c_{2}}{c_{1}}\left(x-3 c_{1}\right) \\
4 c_{1}<x \leq 5 c_{1} & \rightarrow f(x)=2 c_{2}-\frac{c_{2}}{c_{1}}\left(x-4 c_{1}\right) \\
x \geq 5 c_{1} & \rightarrow f(x)=c_{2}
\end{array}\right\}
$$

with, in this case, $c_{1}=0.1$ and $c_{2}=0.5$. The problem is unimodal, but with plateaus.

\section{Deceptive 2 (Comb)}

The search space is $[0,10]$. Function $f$ is defined as: 


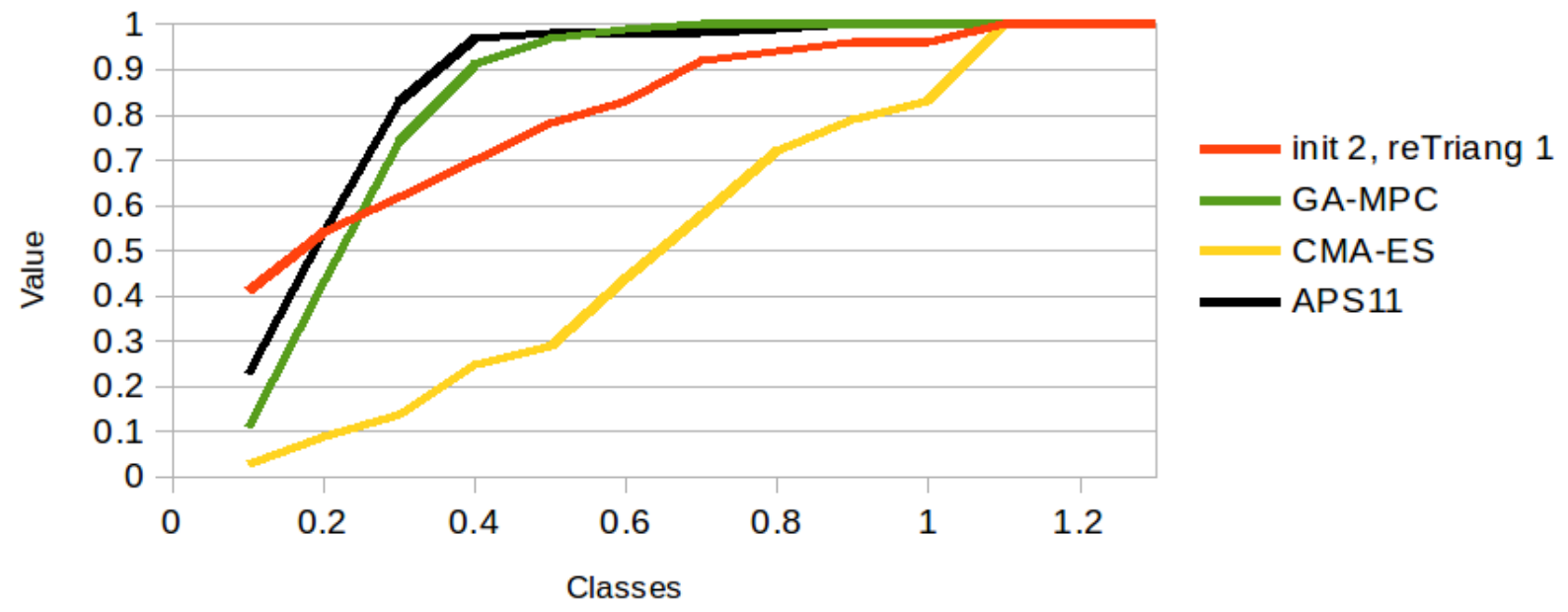

Figure 15: Brush, TMO vs others, 100 runs of 150 evaluations, CDFs curves. On this "negative" NisB problem, TMO is the best method to find good results (smaller than 0.2), but for higher acceptable results, APS11 and even GA-MPC are better. For any demand, CMA-ES is largely the worst.

$$
f(x)=\min \left(c_{2}, 1+\sin \left(c_{1} x\right)+\frac{x}{c_{1}}\right)
$$

with, in this case, $c_{1}=10$ and $c_{2}=1$. The problem is multimodal, but with plateaus.

\section{Deceptive 3 (Brush)}

The search space is $[0,10]^{2}$. Function $f$ is defined as:

$$
f\left(x_{1}, x_{2}\right)=\min \left(c_{2}, \sum_{d=1}^{2}\left|x_{d} \sin \left(x_{d}\right)\right|+\frac{x_{d}}{c 1}\right)
$$

with, in this case, $c_{1}=10$ and $c_{2}=1$. The problem is multimodal and nonseparable.

\section{When we know nothing, the middle is the best choice}

\section{On the search space}

Let $x^{*}$ be the solution point (we do suppose here it is unique). If we sample $x$, the error is $\left\|x-x^{*}\right\|$. At the very beginning, as we know nothing, the probability distribution of $x^{*}$ is uniform on the search space. Roughly speaking, it can be 
anywhere with the same probability. So, we have the sample $x$ in order to minimise the risk given by

$$
r=\int_{x^{*} \in S}\left\|x-x^{*}\right\|
$$

Let us solve it for $D=1$, and $S=\left[x_{\min }, x_{\max }\right]$. We have

$$
\begin{aligned}
r & =\int_{u=x_{\min }}^{x}(x-u) d u+\int_{u=x}^{x_{\max }}(u-x) d u \\
& =\left[x u-\frac{u^{2}}{2}\right]_{u=x_{\min }}^{x}+\left[\frac{u^{2}}{2}-x u\right]_{u=x}^{x_{\max }} \\
& =x^{2}-\left(x_{\max }+x_{\min }\right) x+\frac{x_{\max }^{2}+x_{\min }^{2}}{2}
\end{aligned}
$$

And the minimum of this parabola is given by

$$
x=\frac{x_{\max }+x_{\min }}{2}
$$

For $D>1$ the proof is technically more complicated, but the result is the same: the less risky first point is the centre of the search space.

\section{On the value space}

The same reasoning can be applied to the value space, when we do not make any hypothesis like say a positive local NisB correlation. On any unknown position of the search space the distribution of the possible values on $\left[y_{\min }, y_{\max }\right]$ is uniform and therefore the less risky is, again, the middle, i.e. $\frac{y_{\max }+y_{\min }}{2}$.

\section{Variability of a landscape}

We use here a specific definition, which is different from the definition of variance in probability theory. Let $f$ be a numerical function on the search space $S$. What we call variability on a subspace $s$ of $S$ is the quantity

$$
v=\int_{s^{4}}\left|\frac{f\left(x_{2}\right)-f\left(x_{1}\right)}{\left\|x_{2}-x_{1}\right\|}-\frac{f\left(x_{3}\right)-f\left(x_{1}\right)}{\left\|x_{3}-x_{1}\right\|}\right|
$$

where $\left\{x_{1}, x_{2}, x_{3}\right\}$ is an element of $s^{3}=s \otimes s \otimes s$ (Euclidean product), under the constraint $x_{3}=x_{1}+\lambda\left(x_{2}-x_{1}\right)$ or, equivalently, $\left(x_{2}-x_{1}\right) \times\left(x_{3}-x_{2}\right)=0$ (cross product). The definition may seem to be complicated, but it just means that in any direction the slope of the landscape is constantly the same.

\section{Morphing an estimation of distribution}

There are many possible ways to "distort" a given distribution. In this study we use one based on a simple user-defined numerical parameter. The principle (in case of minimisation) is to temporarily increase/decrease the good (small) 
values, in order to increase/decrease the probability to be selected by a uniform random choice.

A safe way to present it is to give a code. Here is a possible MATLAB ${ }^{\circledR}$ one.

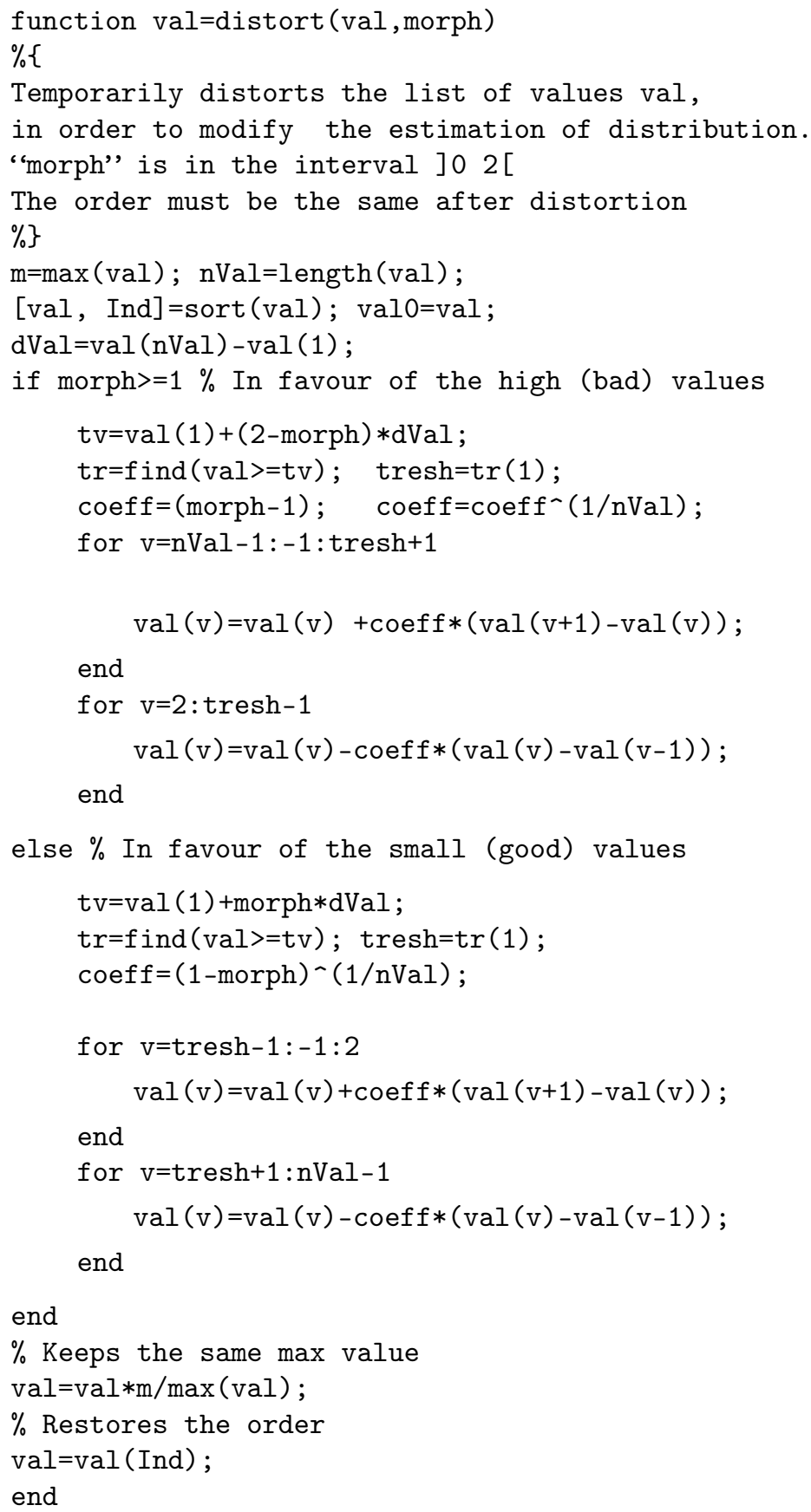




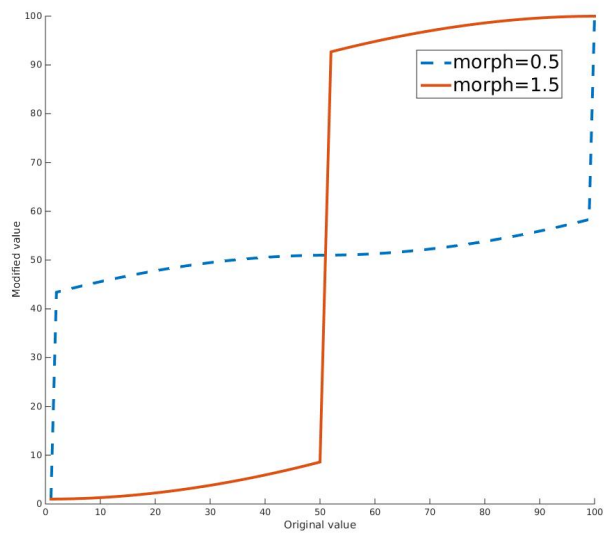

Figure 16: Distortions of a list of values, in case of minimisation. When morph $<1$, good (small) values are temporarily increased, and the contrary for morph $>1$.

The figure 16 shows how the values are temporarily distorted in two cases: optimistic (morph $=0.5)$, when the user thinks that the process can safely be more greedy, or, on the contrary, cautious $(\operatorname{morph}=1.5)$, when the user thinks there is a risk of premature convergence.

The figures 17 present two examples of modified probability distributions We are in the case of minimisation, and the original distribution is the one of the figure $7 \mathrm{~b}$.

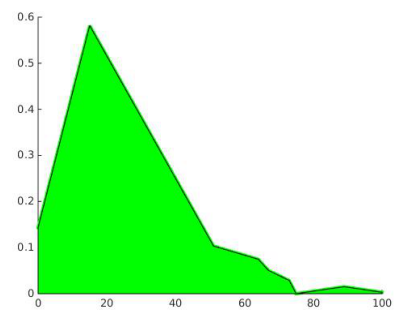

(a) Optimistic morphing

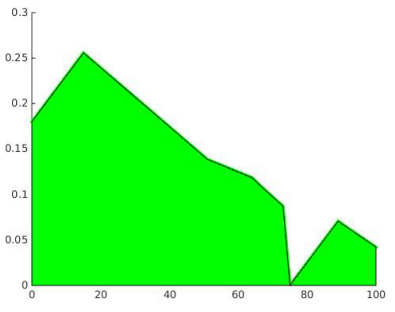

(b) Cautious morphing

Figure 17: Morphing an estimation of distribution.

\section{Signatures}

The concept of signature of an optimiser is presented in [Clerc(2015)]. Basically, a signature is a $2 D$ plot of 10,000 points. To generate them we consider the 
Impossible Plateau problem defined by $f(x)=1$ on $[0,1]^{2}$.

We run 10 times the algorithm, letting it generate 1000 points each time, and we plot all these points on the same figure. For stochastic algorithms two signatures may be different but nevertheless similar, like handwritten personal signatures.

Thanks to this approach, some biases can be detected. The most common ones are a bias in favour of the centre of the search space, and a bias in favour of the borders. Also if the plot seems to be not very dense, it means that many points are (almost) on the same position: they are quasi-duplicates. Note that this last property does not necessarily mean that the algorithm is not efficient. It may just mean that it is able to refine its search "around" a given position (what is often called "exploitation").

On the contrary, centre bias and border bias are often drawbacks, for they mean than some areas may be not well explored. The figure 18 presents the signatures of four methods, including TMO. From this point of view, the worst is CMA-ES, but TMO (at least the basic one) is also slightly concerned by these defaults. 


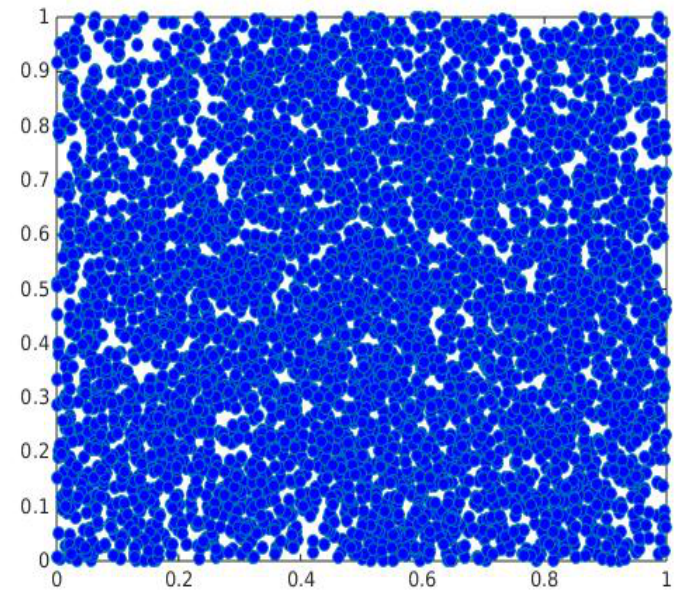

(a) GA-MPC. Almost no quasi-duplicates, no bias.

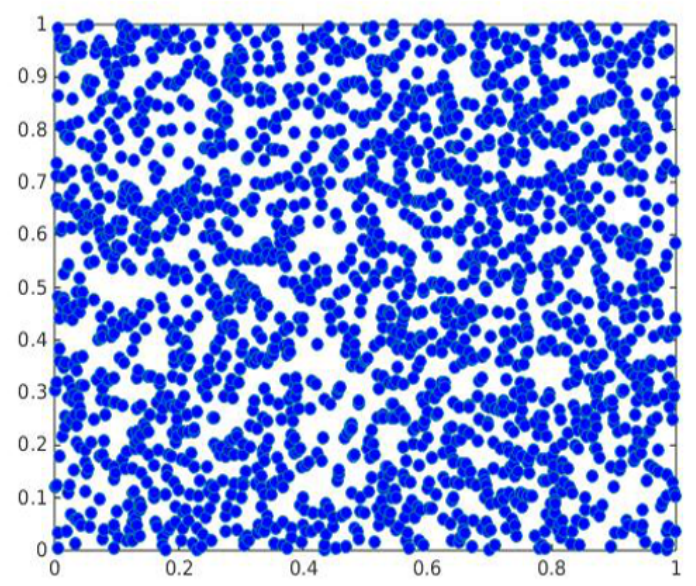

(c) APS. Many quasi-duplicates, no bias.

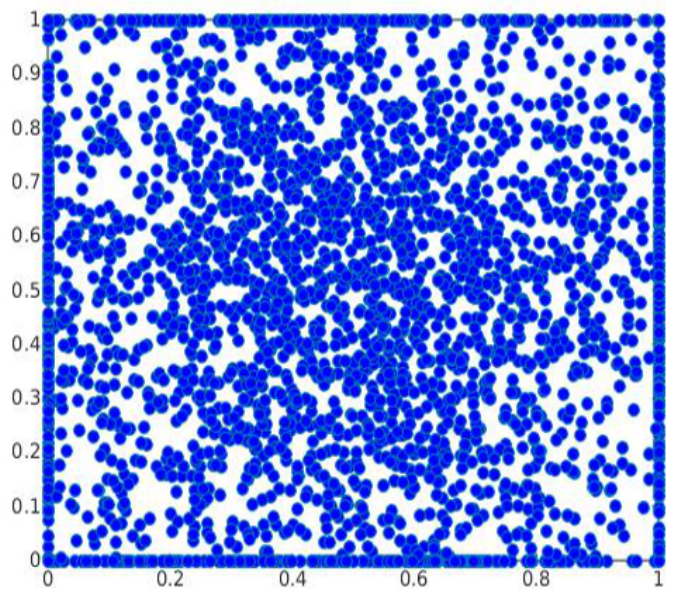

(b) CMA-ES. Many quasi-duplicates, centre bias, high border bias.

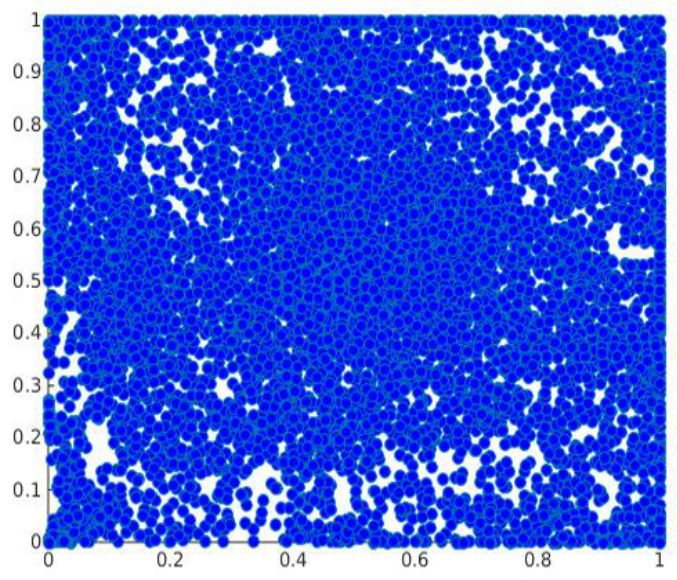

(d) TMO. No duplicates, slight centre bias, slight border bias.

Figure 18: Signatures of four algorithms. 


\section{References}

[Beyhaghi et al.(2015)Beyhaghi, Cavaglieri, and Bewley] Pooriya Beyhaghi, Daniele Cavaglieri, and Thomas Bewley. Delaunay-based derivative-free optimization via global surrogates, part I: linear constraints. Journal of Global Optimization, pages 1-52, November 2015. ISSN 0925-5001, 1573-2916. doi: 10.1007/s10898-015-0384-2.

[Clerc(2007)] Maurice Clerc. When Nearer is Better. 19 pages, 2007. URL https://hal archives-ouvertes .fr/hal-00137320.

[Clerc(2015)] Maurice Clerc. Guided Randomness in Optimization. ISTE (International Scientific and Technical Encyclopedia), Wiley, 2015. ISBN 9781-78548-054-6.

[Elsayed et al.(2011)Elsayed, Sarker, and Essam] Saber M. Elsayed, Ruhul A. Sarker, and Daryl L. Essam. GA with a New Multi-Parent Crossover for Solving IEEE-CEC2011 Competition Problems. 2011.

[Glover and Laguna(1997)] Fred Glover and M. Laguna. Tabu Search. Kluwer Academic Publishers, 1997.

[Han and Zhang(2012)] Zhong-Hua Han and Ke-Shi Zhang. Surrogate-based optimization. INTECH Open Access Publisher, 2012.

[Hansen(2009)] Nikolaus Hansen. The CMA Evolution Strategy: A Tutorial. Technical report, 2009.

[Omran and Clerc(2013)] Mahamed G. H. Omran and Maurice Clerc. An Adaptive Population-based Simplex Method for Continuous Optimization. 2013. 